\title{
Resistance exercise reduces liver fat and its mediators in non-alcoholic fatty liver disease independent of weight loss
}

\author{
Kate Hallsworth, ${ }^{1,2,3}$ Gulnar Fattakhova, ${ }^{2}$ Kieren G Hollingsworth, ${ }^{1,2}$ \\ Christian Thoma, ${ }^{1,2,3}$ Sarah Moore, ${ }^{1,2,3}$ Roy Taylor, ${ }^{1,2}$ Christopher P Day, ${ }^{1,2}$ \\ Michael I Trenell $1,2,3$
}

'Institute of Cellular Medicine, Newcastle University, Newcastle upon Tyne, UK ${ }^{2}$ NIHR Biomedical Research Centre for Ageing and Age-related Disease, Newcastle University, Newcastle upon Tyne, UK

${ }^{3}$ MRC Centre for Brain Ageing and Vitality, Newcastle University, Newcastle upon Tyne, UK

\section{Correspondence to}

Dr Michael Trenell, MoveLab, 4th Floor William Leech Building, Newcastle University, NE1 7RU, UK; michael.trenell@ncl.ac.uk

Revised 25 May 2011 Accepted 27 May 2011 Published Online First 27 June 2011

\section{UNLCKT:}

This paper is freely available online under the BMJ Journals unlocked scheme, see http:// gut.bmi.com/site/about/ unlocked.xhtml

\section{ABSTRACT}

Background Lifestyle interventions focusing on weight loss remain the cornerstone of non-alcoholic fatty liver disease (NAFLD) management. Despite this, the weight losses achieved in research trials are not easily replicated in the clinic and there is an urgent need for therapies independent of weight loss. Aerobic exercise is not well sustained and the effectiveness of the better tolerated resistance exercise upon liver lipid and mediators of liver lipid has not been assessed. Methods Sedentary adults with clinically defined NAFLD were assigned to 8 weeks of resistance exercise $(n=11)$ or continued normal treatment $(n=8)$.

Results 8 weeks of resistance exercise elicited a $13 \%$ relative reduction in liver lipid $(14.0 \pm 9.1$ vs $12.2 \pm 9.0$; $\mathrm{p}<0.05$ ). Lipid oxidation (submaximal RO $\Delta$ $-0.020 \pm 0.010$ vs $-0.004 \pm 0.003 ; p<0.05)$, glucose control $(-12 \%$ vs $+12 \%$ change AUC; $p<0.01)$ and homeostasis model assessment insulin resistance $(5.9 \pm 5.9$ to $4.6 \pm 4.6$ vs $4.7 \pm 2.1$ to $5.1 \pm 2.5 ; p<0.05)$ were all improved. Resistance exercise had no effect on body weight, visceral adipose tissue volume, or whole body fat mass ( $p>0.05$ ).

Conclusion This is the first study to demonstrate that resistance exercise specifically improves NAFLD independent of any change in body weight. These data demonstrate that resistance exercise may provide benefit for the management for non-alcoholic fatty liver, and the long-term impact of this now requires evaluation.

Non-alcoholic fatty liver disease (NAFLD) represents a spectrum from asymptomatic steatosis to potentially life-threatening non-alcoholic steatohepatitis, with an overall prevalence of NAFLD in western countries of $20-30 \%{ }^{1}$ Patients with simple steatosis have a relatively benign 'liver' prognosis with a $1-2 \%$ risk of developing clinical evidence of cirrhosis over 15-20 years. Patients with non-alcoholic steatohepatitis and fibrosis can progress to cirrhosis at a rate of approximately $12 \%$ over 8 years. ${ }^{2}$ Once cirrhosis develops, patients are at a high risk of developing hepatic decompensation and of dying from a liver-related cause.

To date, weight loss is the only confirmed therapy for the treatment of NAFLD, and lifestyle interventions remain the cornerstone of management. $^{1}{ }^{2}$ Thiozolidinedione therapy has shown promise in providing a temporary reduction in liver fat, ${ }^{34}$ although progressive weight gain, side effects and cost have prevented them from being used in

\section{Significance of this study}

What is already known about this subject?

- Lifestyle interventions incorporating weight loss improve liver fat in NAFLD.

- Weight loss is difficult to achieve in clinical practice.

- Evidence on the effect of exercise on liver fat is insufficient, limiting the effective use of exercise in clinical care.

What are the new findings?

- Resistance exercise reduces liver fat independent of weight loss in people with NAFLD.

- Resistance exercise improves fat oxidation independent of weight loss in people with NAFLD.

- Resistance exercise improves glucose control independent of weight loss in people with NAFLD.

How might it impact on clinical practice in the foreseeable future?

- Helping people achieve and sustain a physically active lifestyle is one of the most pressing clinical needs for NAFLD management.

- This study demonstrates that resistance exercise holds clinical benefits, independent of weight loss, and provides an alternative to aerobic exercise.

- The lower cardiorespiratory demand may also make resistance exercise more accessible to patients than aerobic exercise, improving longterm sustainability.

routine care. Lifestyle interventions have been shown to reduce markers of liver lipid and metabolic control ${ }^{5-7}$ in addition to reducing intrahepatic lipid (IHL) ${ }^{8-11}$ However, weight loss is difficult to achieve and sustain. Physical activity and exercise in NAFLD management could potentially be effective in decreasing IHL. Cross-sectional studies have shown that higher levels of physical activity are associated with lower levels of IHL. ${ }^{5} 1213$ To date, two studies have reported a beneficial effect of aerobic exercise regimes upon liver function, independent of weight loss. ${ }^{14}{ }^{15}$ However, the use of aerobic exercise may not be optimal as the high 
cardiorespiratory demand is associated with fatigue and discomfort, and long-term compliance is poor. Population-based studies ${ }^{13}$ suggest that resistance exercise is associated with lower levels of IHL. Resistance exercise has a lower cardiorespiratory demand and is associated with similar metabolic benefits. There have been no previous studies of the direct effect of resistance exercise upon IHL and metabolism, even though it has clear advantages in terms of acceptability and sustainability. ${ }^{16} 17$

The primary aim of this study was to determine the effect of resistance exercise without weight loss, on IHL in adults with NAFLD. The secondary aims were to determine the effect of resistance exercise on mediators of IHL; glucose tolerance and insulin sensitivity, fat oxidation, abdominal adiposity and body composition.

\section{EXPERIMENTAL PROCEDURES}

Twenty-eight people with NAFLD were screened for this study. Seven were excluded after screening (two transpired to be taking insulin for their diabetes; one was excluded due to an abdominal hernia; four due to $<5 \%$ IHL on baseline MRI). Twenty-one sedentary ( $\leq 60$ min vigorous activity per week) adults with clinically defined non-advanced NAFLD were therefore randomly assigned to either exercise $(n=11)$ or standard care $(n=10)$. The study was powered to detect a $2.02 \%$ absolute change $(\Delta)$ in intrahepatic lipid between the treatment and control groups (SD $2.8 \%, \alpha 5 \%$ and $\beta 50 \%$ ), based on changes reported from an aerobic exercise study in NAFLD. ${ }^{14}$ Non-advanced NAFLD was defined as greater than $5 \%$ IHL and a score of less than -1.445 on the NAFLD fibrosis scoring system, ${ }^{18}$ which indicates a lower percentage chance of having stage $3 / 4$ fibrosis. General descriptions can be found in table 1. Exclusion criteria included heart or kidney disease, implanted ferrous metal, pre-existing medical conditions preventing participation in the exercise programme, insulin sensitising treatment or dietary change (for people with type 2 diabetes mellitus, diet and metformin were acceptable for inclusion if stable for 6 months) and alcohol intake above 21 units for men or 14 units for women. Subjects would be excluded from analysis if body weight changed more than $2.5 \%$ from baseline during the study as this could have an independent effect on IHL. The study protocol was approved by County Durham and Tees Valley 2 Research Ethics Committee. All participants provided written informed consent. Following an initial screening visit, glucose control, lipid oxidation, abdominal lipid depots and liver lipid were measured at baseline and after the 8-week intervention.

\section{Progressive exercise test/screening visit}

At baseline, a medical history, full physical examination and progressive exercise test were used to screen for undiagnosed cardiac disease. Resting 12-lead ECG (Custo med GmbH, Ottobrunn, Germany) and blood pressure (Suntech Tango+, Suntech Medical Ltd, Oxford, UK) were measured, in a seated position, to determine normal cardiac function. Peak oxygen consumption was determined using an electronically braked recumbent cycle ergometer (Corival Lode BV, Groningen, The Netherlands). Following a 5 min warm up at $25 \mathrm{~W}$, resistance was increased by $1 \mathrm{~W}$ per $8 \mathrm{~s}$ until the participant could no longer maintain a cadence of $60 \mathrm{rpm}$, chose to stop, or continuing was contraindicated. The ECG was used to monitor heart rhythm continuously and blood pressure was measured every 2 min during the exercise test. Expired gases were collected using a Hans Rudolf breathing mask and analysed online for oxygen consumption, carbon dioxide elimination and ventilation (CORTEX Biophysik, Leipzig, Germany).

\section{Physical activity}

Physical activity and energy expenditure were assessed objectively using a validated ${ }^{19}$ multisensor array (SenseWear $\mathrm{PrO}_{3}$, Bodymedia Inc, Pennsylvania, USA) worn on the right upper arm for 7 days before random assignment and for the final 7 days of the intervention. The armband provided estimates of daily energy expenditure, average metabolic equivalents, sedentary time, duration and intensity of physical activity, number of steps, sleep duration and duration armband worn.

\section{Anthropometry}

Body weight and body composition were measured using an electronic scale and air displacement plethysmography (BodPod, Life Measurement Inc, California, USA). ${ }^{20-22}$ Height was measured with a stadiometer (SECA 799; SECA, Birmingham, UK). Waist circumference was measured at the midpoint between the lower costal margin and the level of the anterior superior iliac crests. Hip circumference was measured at the level of the greater trochanter.

\section{Liver and abdominal fat measurement}

Magnetic resonance studies were performed using a 3 Tesla Philips Achieva scanner (Philips Medical Systems, Best, The Netherlands). Following an $8 \mathrm{~h}$ fast, IHL was measured by localised ${ }^{1} \mathrm{H}$-MRS (PRESS, TR/TR $3000 \mathrm{~ms} / 35 \mathrm{~ms}, 3 \times 3 \times 3 \mathrm{~cm}$

Table 1 Subject characteristics

\begin{tabular}{|c|c|c|c|c|c|c|c|}
\hline & \multicolumn{3}{|l|}{ Control } & \multicolumn{3}{|l|}{ Exercise } & \multirow{2}{*}{$\begin{array}{l}\text { Time } \times \text { treatment } \\
\text { interaction ( } p \text { value) }\end{array}$} \\
\hline & Baseline & Post-treatment & $\overline{\text { p Value }}$ & Baseline & Post-treatment & $\overline{\text { p Value }}$ & \\
\hline \multicolumn{8}{|l|}{ Anthropometry } \\
\hline BMI $\left(\mathrm{kg} / \mathrm{m}^{2}\right)$ & $32.3(4.8)$ & $32.5(4.2)$ & 0.42 & $32.3(4.9)$ & $32.3(4.5)$ & 0.89 & 0.43 \\
\hline Weight (kg) & $94.0(12.0)$ & $94.6(10.7)$ & 0.37 & $96.1(10.9)$ & $96.1(10.5)$ & 0.99 & 0.44 \\
\hline Waist circumference $(\mathrm{cm})$ & $108(9)$ & $110(7)$ & 0.42 & $107(11)$ & $109(12)$ & 0.32 & 0.55 \\
\hline Hip circumference (cm) & $110(6)$ & $115(9)$ & 0.06 & $110(9)$ & $111(9)$ & 0.85 & 0.13 \\
\hline Waist to hip ratio & $0.99(0.05)$ & $0.96(0.06)$ & 0.16 & $0.97(0.06)$ & $0.98(0.06)$ & 0.21 & 0.15 \\
\hline $\mathrm{VO}_{2 \text { peak }}(\mathrm{ml} / \mathrm{kg}$ per $\mathrm{min})$ & $18.5(5.2)$ & - & - & $21.8(3.8)$ & - & - & - \\
\hline \multicolumn{8}{|l|}{ Metabolic } \\
\hline $\operatorname{ALT}(\mathrm{U} / \mathrm{l})$ & $61.6(41.4)$ & $61.4(44.0)$ & 0.92 & $59.6(38.6)$ & $59.6(39.0)$ & 0.99 & 0.98 \\
\hline Total cholesterol (mmol/l) & $4.5(1.1)$ & $4.6(0.9)$ & 0.47 & $5.0(1.8)$ & $5.0(1.7)$ & 0.67 & 0.46 \\
\hline Triglyceride (mmol/l) & $1.46(0.61)$ & $1.50(0.75)$ & 0.81 & $1.79(0.72)$ & $1.62(0.55)$ & 0.21 & 0.30 \\
\hline $\mathrm{HbA1c}$ & $6.5(1.1)$ & $6.8(1.8)$ & 0.40 & $6.1(0.8)$ & $6.0(0.7)$ & 0.17 & 0.24 \\
\hline
\end{tabular}

\footnotetext{
Values are means $(S D) ; n=19$

There are no statistical differences between groups or with treatment in these measures.
}

$\mathrm{BMI}$, body mass index; $\mathrm{HbA1c}$, haemoglobin $\mathrm{A} 1 \mathrm{c}$; $\mathrm{VO}_{2 \text { peak, }}$ aerobic capacity. 
voxel, SENSE torso array). Blinded quantification of the spectra (water and $\mathrm{CH}_{2}$ resonances) was performed using the java-based magnetic resonance user interface (jMRUI version 3.0). ${ }^{23} 24$ Following manual first and second order phase correction, spectra were analysed using a non-linear least squares algorithm (AMARES). ${ }^{25}$ IHL was expressed as a percentage of liver volume, corrected for proton density of water and lipid. ${ }^{26}$

Subcutaneous and visceral fat content was performed by acquiring images at the L4/L5 junction using a three-point Dixon sequence (TR/TE/number of averages/flip angle $50 \mathrm{~ms} /$ $3.45,4.60,5.75 \mathrm{~ms} / 1 / 30^{\circ}$, matrix $160 \times 109$, median field of view (FOV) $440 \mathrm{~mm}$, range $400-480 \mathrm{~mm}$ to suit subject size with $70 \%$ phase FOV). The slice was acquired during a breath-hold and with slice thickness of $10 \mathrm{~mm} .^{27} 28$ Fat and water were separated, and binary gating applied to produce a map of structures containing more than $50 \%$ fat, identified as subcutaneous and visceral fat. A watershed algorithm was used to divide the binary image into distinct areas and allowed easy separation of the subcutaneous and visceral fat. Image ${ }^{29}$ was used to subtract the two areas to produce the area of visceral fat.

\section{Glucose control}

Following an $8 \mathrm{~h}$ overnight fast a cannula was inserted into a forearm vein. A $75 \mathrm{~g}$ glucose load (Lucozade Original; Glaxo SmithKline, Brentford, UK) was consumed within $5 \mathrm{~min}$. Blood samples were taken at times $0,5,10,15,20,30,40,50,60,75,90$ and $120 \mathrm{~min}$. Samples were analysed for whole blood glucose (YSI 2300 Stat Plus-D; Yellow Springs Instruments, Yellow Springs, Ohio, USA), plasma non-esterified fatty acid (NEFA) (NEFA-HA; Wako Ltd, Osaka, Japan) and plasma insulin (CoatA-Count Insulin RIA kit; Diagnostic Products Corporation, California, USA). The area under the curve for the resulting glucose response profile was calculated using the trapezoidal rule $^{30}$ and insulin resistance determined using the homeostasis model assessment insulin resistance (HOMA-IR). ${ }^{31}$ NEFA suppression was assessed during the frequently sampled oral glucose tolerance test (fsOGTT) and the $0-30$ min change used as a measure of NEFA suppression. ${ }^{32}$

Fasting samples were also analysed in a clinical pathology accredited laboratory (Newcastle Upon Tyne Hospital NHS Foundation Trust, Department of Clinical Biochemistry) for: ALT, total cholesterol, triacylglycerols and haemoglobin A1c (HbA1c). Serum samples were collected in silica clot activator polymer gel containing vacutainers (BD Diagnostics, Plymouth, UK) - total cholesterol, triacylglycerols and ALT were measured using a Roche Modular P and test kits (Roche Diagnostics Ltd, Burgess Hill, UK), respectively. HbA1c was measured using a TOSOH HLC-723G7 (Tosoh Corporation, Tokyo, Japan).

\section{Lipid oxidation at rest and submaximal exercise}

Resting substrate oxidation was determined by expired gas analysis using a Hans Rudolf breathing mask while participants lay supine for $30 \mathrm{~min}$ in a quiet room. The first $15 \mathrm{~min}$ were an acclimatisation period and the second $15 \mathrm{~min}$ were used to determine resting substrate oxidation. Following a 5-min warm up on the recumbent cycle ergometer at $25 \mathrm{~W}$, resistance was increased to $50 \%$ of the maximal oxygen uptake determined at screening and maintained for $60 \mathrm{~min}$. Expired air was collected every $15 \mathrm{~min}$. Respiratory quotient was calculated from oxygen consumption/carbon dioxide elimination.

\section{Study intervention}

Resistance exercise was performed three times per week on nonconsecutive days for 8 weeks. The programme consisted of eight exercises: biceps curl; calf raise; triceps press; chest press; seated hamstrings curl; shoulder press; leg extension and lateral pull down (Precor, Woodinville, USA). Each session lasted between 45 and $60 \mathrm{~min}$ and consisted of a $10 \mathrm{~min}$ warm-up at approximately $60 \%$ maximum heart rate on a cycle ergometer followed by resistance exercise done as a circuit, ending with a repeat of the warm-up described. The one repetition maximum was measured $^{33}$ at baseline and following the intervention. Initially, participants did two circuits using $50 \%$ of their one repetition maximum, progressing to three circuits, using a minimum $70 \%$ of their one repetition maximum by week 7 . Participants were encouraged to increase the resistance used each week when possible. Biweekly supervised sessions were used to encourage adherence and progression and to resolve any problems. Heart rate was recorded during each session (Polar RS400; Polar Electro Oy, Kempele, Finland) and was used alongside exercise logs to assess adherence.

\section{Statistics}

Following tests for normal distribution, between-group differences were evaluated using an unpaired t-test and within-group differences using a paired t-test (two way). Treatment group $\times$ time interactions were assessed using a two-way analysis of variance. Analyses were performed using Minitab version 15 (Minitab Inc., State College, Pennsylvania, USA). Statistical significance was set at $p<0.05$. Data are mean $\pm S D$ unless otherwise stated.

\section{RESULTS}

No subjects withdrew during the trial and all subjects allocated to the exercise group completed all 24 sessions of resistance exercise training. Two subjects (controls) were excluded from analysis (one individual lost $>5 \%$ of his body weight during the 8-week period and one had a change in his diabetes medication). Nineteen subjects (eight control; 11 exercise) completed the study. The groups were well matched for weight, body mass index and waist/hip circumference (table 1). The exercise group were younger (52 \pm 13.3 ; range $33-72$ years) compared with the control group (62 \pm 7.4 ; range $51-71$ years; $p=0.05$ ). There was no correlation between age and change in IHL $(\mathrm{p}>0.05)$.

\section{Anthropometry and body composition}

Body mass index remained constant in both groups during the study $\left(32 \pm 4.9\right.$ to $32 \pm 4.5$ vs $32 \pm 4.8$ to $32 \pm 4.2 \mathrm{~kg}$. $\mathrm{m}^{2}$ in exercise and control). There were no significant changes in weight, waist or hip circumference, waist to hip ratio, body composition, visceral or subcutaneous fat in either group (see tables 1 and 2).

\section{Intrahepatic lipid}

Resistance exercise elicited a $13 \%$ relative reduction in IHL with no change in the control $(\mathrm{p}<0.01$; table 2 , figure $1 \mathrm{~A})$. There was a significant time by treatment interaction for resistance exercise $(\mathrm{p}<0.05$; table 2 , figure 1A) although the significant fall in IHL did not achieve a significant difference between the two groups at the end of the intervention. However, three of the participants in the exercise group moved from having clinically significant NAFLD to being within normal limits ( $<5 \%$ IHL). No control subject moved into the normal liver fat range. The change in IHL with both groups combined was weakly associated with the change in the number of steps walked per day $\left(R^{2}=0.28, p<0.05\right)$, but not with active energy expenditure $\left(\mathrm{R}^{2}=0.06, \mathrm{p}>0.05\right)$. 
Table 2 Intrahepatic lipid concentration, subcutaneous and visceral adipose tissue, body composition, glucose control, insulin sensitivity, NEFA suppression index and substrate oxidation during submaximal exercise

\begin{tabular}{|c|c|c|c|c|c|c|c|}
\hline & \multicolumn{3}{|l|}{ Control } & \multicolumn{3}{|l|}{ Exercise } & \multirow{2}{*}{$\begin{array}{l}\text { Time } \times \text { treatment } \\
\text { interaction ( } p \text { value }\end{array}$} \\
\hline & Baseline & Post-treatment & p Value & Baseline & Post-treatment & p Value & \\
\hline Intrahepatic lipid (\%) & $11.2(8.4)$ & $11.5(7.4)$ & 0.80 & $14.0(9.1)$ & $12.2(9.0)$ & $0.01^{*}$ & $0.05 \dagger$ \\
\hline Visceral adipose tissue $\left(\mathrm{cm}^{2}\right)$ & $2558(715)$ & $2445(644)$ & 0.31 & $2098(809)$ & $2165(816)$ & 0.26 & 0.15 \\
\hline Subcutaneous adipose tissue $\left(\mathrm{cm}^{2}\right)$ & $3512(970)$ & $3574(936)$ & 0.30 & $3275(1165)$ & $3221(1180)$ & 0.46 & 0.21 \\
\hline Fat mass (\% body mass) & $41(6)$ & $41(7)$ & 0.88 & $37(8)$ & $36(8)$ & 0.12 & 0.38 \\
\hline Fasting glucose $(\mathrm{mmol} / \mathrm{l})$ & $5.9(2.3)$ & $6.4(3.3)$ & 0.33 & $6.0(2.1)$ & $5.2(0.9)$ & 0.08 & 0.06 \\
\hline Fasting insulin $(\mathrm{pmol} / \mathrm{l})$ & $18.14(4.52)$ & $18.97(9.82)$ & 0.18 & $20.55(13.68)$ & $18.64(13.25)$ & 0.18 & 0.33 \\
\hline Fasting NEFA $(\mu \mathrm{mol} / \mathrm{l})$ & $0.48(0.13)$ & $0.50(0.17)$ & 0.62 & $0.44(0.19)$ & $0.43(0.13)$ & 0.77 & 0.56 \\
\hline HOMA-IR & $4.7(2.1)$ & $5.1(2.5)$ & 0.57 & $5.9(5.9)$ & $4.6(4.6)$ & $0.05 \ddagger$ & 0.06 \\
\hline fsOGTT, AUC & $839(299)$ & $940(421)$ & 0.15 & $885(269)$ & $777(184)$ & $0.01^{*}$ & $0.01 \S$ \\
\hline NEFA-S (0-30 min of fsOGTT) & $0.07(0.10)$ & $-0.02(0.10)$ & 0.15 & $0.00(0.10)$ & $0.00(0.14)$ & 0.99 & 0.25 \\
\hline Resting R0 & $0.86(0.09)$ & $0.86(0.06)$ & 0.76 & $0.86(0.05)$ & $0.86(0.06)$ & 0.92 & 0.76 \\
\hline Submaximal exercise $\mathrm{R} 0$ & $0.90(0.02)$ & $0.89(0.02)$ & 0.08 & $0.93(0.02)$ & $0.91(0.01)$ & $0.02 \ddagger$ & 0.05 \\
\hline
\end{tabular}

Values are means (SD); $n=19$.

*Significant difference baseline versus post-treatment $(p<0.01)$

$\dagger$ Significant difference treatment $\times$ time interaction $(p<0.05)$.

$\ddagger$ Significant difference baseline versus post-treatment $(p<0.05)$.

$\S$ Significant difference treatment $\times$ time interaction $(p<0.01)$.

AUC, area under the curve; fsOGTT, frequently sampled oral glucose tolerance test; HOMA-IR, homeostasis model of insulin resistance; NEFA-S, non-esterified fatty acid suppression index $\mathrm{RO}$, respiratory quotient.

\section{Blood lipids and liver enzymes}

There were no significant changes in blood lipids or ALT in either group (table 1).

\section{Glucose control}

The exercise group demonstrated improved glucose control after 8 weeks as indicated by a decrease in glucose area under the curve during the fsOGTT compared with control $(p<0.01$; figure $1 \mathrm{~B}$; table 2). The exercise group showed a significant improvement in insulin sensitivity after 8 weeks, as demonstrated by a decrease in HOMA-IR $(5.9 \pm 5.9$ to $4.6 \pm 4.6$ vs $4.7 \pm 2.1$ to $5.1 \pm 2.5 ; \mathrm{p}<0.05$; table 2 ), although time by treatment interaction for resistance exercise and insulin resistance just missed statistical significance $(p=0.055)$. Fasting insulin levels remained relatively unchanged in both groups (table 2). Fasting glucose levels were reduced in the exercise group after the intervention compared with the control but this was not statistically significant $(6.0 \pm 2.1$ to $5.2 \pm 0.9$ vs $5.9 \pm 2.3$ to $6.4 \pm 3.3 \mathrm{mmol} / \mathrm{l}$; $\mathrm{p}=.086)$. Time by treatment interaction for resistance exercise and change in fasting glucose was just outside statistical significance $(p=0.06)$. HbA1c remained relatively unchanged in both groups (table 1 ).

\section{NEFA suppression}

Fasting plasma NEFA remained unchanged by the intervention. Similarly, the extent of NEFA suppression during the fsOGTT remained constant in both groups (table 2).

\section{Fat oxidation}

Resistance exercise brought about an increase in fat oxidation during the submaximal exercise test (respiratory quotient $0.93 \pm 0.02$ to $0.90 \pm 0.01$ vs $0.90 \pm 0.02$ to $0.89 \pm 0.02, p<0.05$; $\mathrm{p}<0.05$ time by treatment interaction; table 2; figure $1 \mathrm{C})$. Resting fat oxidation remained constant in both groups (table 2).

\section{Physical activity}

There was a wide range of habitual daily activity (number of steps taken daily measured by Sensewear) in both groups at baseline (range 5046-12479 exercise, mean 8692; range 2781-9159 control, mean 5682). The total number of steps taken on a daily basis was significantly higher in the exercise group post-intervention when compared with the controls $(9848 \pm 3338$ vs $5883 \pm 3073 ; p=0.028)$.

\section{DISCUSSION}

This is the first study to examine the effects of resistance exercise on IHL and its mediators in adults with NAFLD. An 8-week resistance exercise programme brought about an approximately $13 \%$ reduction in liver fat. This was accompanied by an approximately $12 \%$ increase in insulin sensitivity, and increased fat oxidation during submaximal exercise in the absence of any change in body weight.

Although lifestyle modification combining dietary change and exercise produces a robust reduction in IHL, ${ }^{1}$ the data on exercise alone is less definitive. We demonstrated that resistance exercise without weight change is effective in reducing IHL in people with NAFLD. Resistance exercise provides an alternative to aerobic exercise; it improves muscular strength, muscle mass and metabolic control, safely and effectively, in vulnerable populations independent of weight loss. ${ }^{16}$ It places less of a demand on the cardiorespiratory system and may therefore be accessible to more patients. ${ }^{17}$ All participants completed the 8-week programme, showing good adherence and tolerance.

To date, only two studies have reported the effect of exercise alone on liver health in adults with NAFLD, both having used aerobic exercise, but only one reported a direct measure of IHL. A 4-week aerobic exercise intervention showed a similar absolute reduction in IHL $\left({ }^{1} \mathrm{H}-\mathrm{MRS}\right)$ as seen during the present study. The aerobic exercise brought about a reduction in IHL from $8.6 \%$ to $6.8 \% .{ }^{14}$ A 12 -week intervention reported a $47 \%(47 \mathrm{U} / \mathrm{l})$ and $48 \%(30 \mathrm{U} / \mathrm{l})$ reduction in ALT and AST, respectively. ${ }^{15}$ However, these biomarkers can be elevated in the absence of excess liver fat and within the normal range in the presence of elevated liver fat, making them poor indicators for IHL. ${ }^{34-36}$ Interestingly, the present study is the first to monitor habitual physical activity alongside exercise therapy. These data suggest that changes in physical activity habits and the brief aerobic warm up accompanying resistance exercise contributed to the change in IHL only to a small extent. Although the changes in IHL following exercise therapy are significant, the absolute change $(\sim 2 \%$ IHL) 

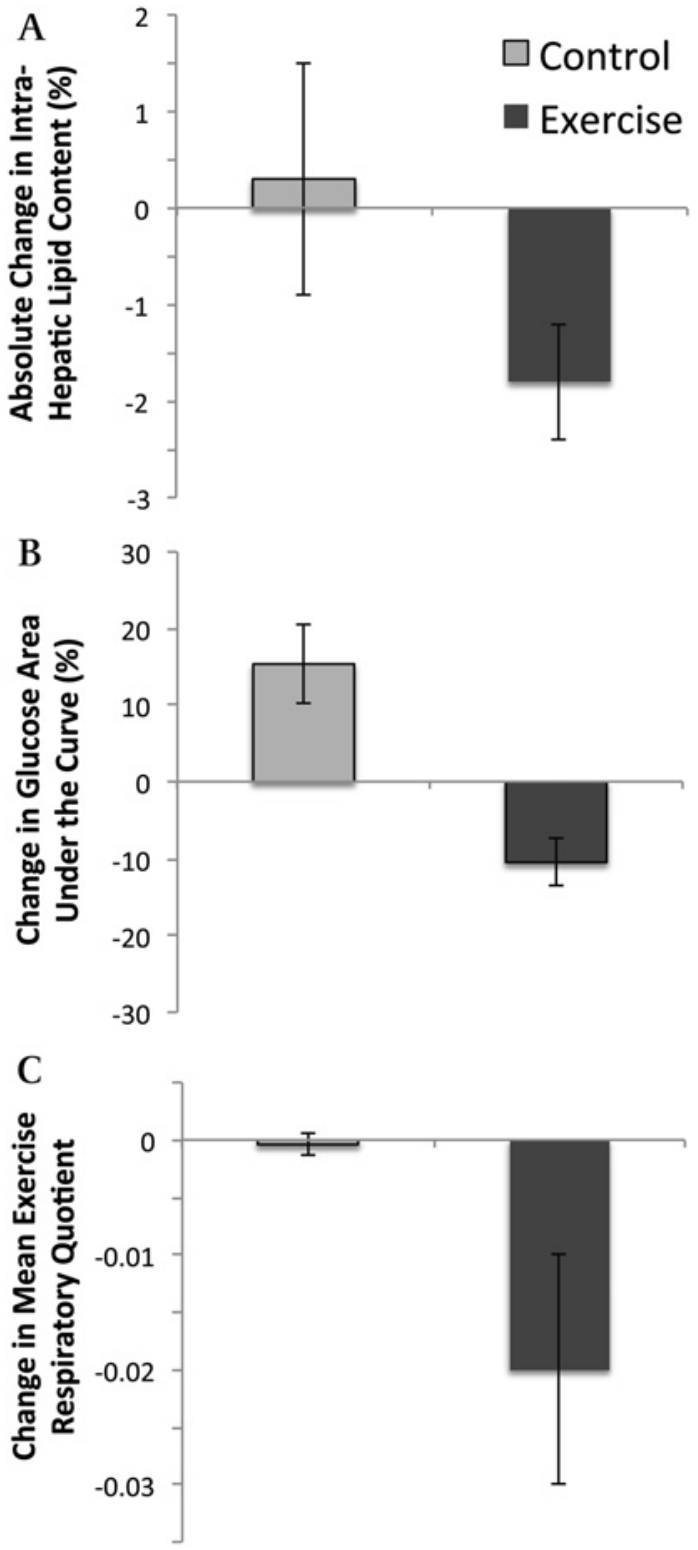

Figure 1 Effect of 8 weeks resistance exercise training (Exercise) or continued standard care (Control) on intrahepatic lipid (A), glucose control from the frequently sampled oral glucose tolerance test $(B)$ and respiratory quotient during submaximal exercise $(\mathrm{C})$; values are means \pm SE. ${ }^{*}$ Significantly different from control $(p<0.05)$.

**Significantly different from control $(p<0.01)$.

was modest compared with the approximately $10 \%$ reduction reported after an $8 \mathrm{~kg}$ weight loss from caloric restriction. ${ }^{37}$ Therefore, the clinical value of exercise in NAFLD appears likely to be as an adjunct to caloric restriction.

Our observed improvement in glucose control is consistent with findings in populations with impaired glucose control or type 2 diabetes mellitus. ${ }^{38} 39$ The aerobic exercise studies in NAFLD have either reported no change ${ }^{14}$ or not reported measures of glucose control. ${ }^{15}$

We observed a pure exercise effect on IHL, which did not involve any change in visceral fat in the patients. There is increasing evidence that the two depots are not mechanistically linked but both tend to reflect adiposity. ${ }^{3}$ Recent findings from the Framingham Heart Study, ${ }^{40}$ and a much smaller cohort, ${ }^{41}$ show IHL to be associated with dyslipidaemia and dysglycaemia independently of visceral fat. Our observation of decreased IHL in the absence of any observable change in visceral or subcutaneous fat provides further information on the separate regulation of IHL and visceral fat.

The mechanisms underlying the change in IHL following exercise are likely to reflect changes in energy balance, circulatory lipids and insulin sensitivity. Insulin sensitivity plays a significant role in IHL homeostasis. High levels of circulatory insulin upregulate SREBP-1c and ChREBP expression in the liver, ${ }^{42}{ }^{43}$ stimulating de novo lipogenesis and increasing IHL. In healthy normoglycaemic humans, hepatic de novo lipogenesis contributes approximately $5 \%$ and $18-23 \%$ of IHL in the fasted and postprandial states, respectively. ${ }^{44}$ Whereas de novo lipogenesis is constantly elevated in those with NAFLD contributing approximately $26 \%$ of IHL irrespective of feeding state, ${ }^{45}$ elevated circulating triacylglycerols exacerbate this problem by impeding insulin-stimulated glucose uptake. ${ }^{46}$ This creates a vicious cycle in which elevated IHL levels impede hepatic insulin action, causing increased portal insulin levels and further increasing IHL. ${ }^{47}$

Our findings suggest that the introduction of resistance exercise breaks this cycle by improving glucose control and fat oxidation. Our observations would support other reports that resistance exercise increases whole-body glucose disposal ${ }^{48}$ at least partly due to increases in skeletal muscle GLUT4, glycogen synthase expression and activity, insulin receptor and glycogen storage. ${ }^{49}$ Skeletal muscle in the resistance exercising individual can thus act to sequester circulating fatty acids and glucose safely, reducing the impact of insulin-stimulated de novo lipogenesis in the liver. Aerobic exercise has been shown to increase intramyocellular triacylglycerol synthesis, ${ }^{50} 51$ while decreasing the accumulation of fatty acid metabolites and suppressing the proinflammatory state associated with insulin resistance. ${ }^{52}$ It remains to be determined whether exercise has any direct transcriptional effects on the liver. Furthermore, as patients with fibrosis were excluded, the benefits of resistance exercise upon the progression of liver disease versus metabolic control cannot be disentangled. It is also important to highlight that IHL is part of the early adaptive response to stress rather than a first hit in disease progression. As such, IHL represents a biomarker of NEFA flux, oxidative, endoplasmic reticulum (ER) and cytokine-mediated stress that results in steatosis and progressive liver damage. ${ }^{53}$

In conclusion, well-tolerated resistance exercise reduced IHL, increased insulin sensitivity, and improved metabolic flexibility in NAFLD independent of weight loss. The absolute effect of resistance exercise studied in isolation was modest but similar to that of aerobic exercise. The benefits of resistance exercise combined with caloric restriction in the clinical management of NAFLD will depend upon long-term maintenance and sustainability of exercise-this now needs to be investigated.

Funding The research leading to these results has received funding from the European Union Seventh Framework Programme (FP7/2007-2013) under grant agreement no Health-F2-2009-241762, for the project FLIP; the Medical Research Council; the UK National Institute for Health Research Biomedical Research Centre on Ageing and Age-Related Diseases and Diabetes UK.

\section{Competing interests None.}

Ethics approval This study was conducted with the approval of the County Durham and Tees Valley 2 Research Ethics Committee.

Provenance and peer review Not commissioned; externally peer reviewed.

\section{REFERENCES}

1. Harrison SA, Day CP. Benefits of lifestyle modification in NAFLD. Gut 2007; 56:1760-9. 
2. Day CP. Non-alcoholic fatty liver disease: current concepts and management strategies. Clin Med 2006;6:19-25.

3. Ravikumar B, Gerrard J, Dalla Man C, et al. Pioglitazone decreases fasting and postprandial endogenous glucose production in proportion to decrease in hepatic triglyceride content. Diabetes 2008;57:2288-95

4. Bajaj M, Suraamornkul S, Pratipanawatr T, et al. Pioglitazone reduces hepatic fat content and augments splanchnic glucose uptake in patients with type 2 diabetes. Diabetes 2003; 52:1364-70.

5. St George A, Bauman A, Johnston A, et al. Independent effects of physical activity in patients with nonalcoholic fatty liver disease. Hepatology 2009;50:68-76.

6. Promrat K, Kleiner DE, Niemeier $\mathbf{H}$, et al. Randomized controlled trial testing the effects of weight loss on nonalcoholic steatohepatitis. Hepatology 2010:51:121-9.

7. Kantartzis $\mathbf{K}$, Thamer C, Peter A, et al. High cardiorespiratory fitness is an independent predictor of the reduction in liver fat during a lifestyle intervention in non-alcoholic fatty liver disease. Gut 2008;58:1281-8.

8. Finucane $\mathbf{F}$, Sharp S, Purslow L, et al. The effects of aerobic exercise on metabolic risk, insulin sensitivity and intrahepatic lipid in healthy older people from the Hertfordshire Cohort Study: a randomised controlled trial. Diabetologia 2010;53:624-31.

9. Goodpaster BH, Katsiaras A, Kelley DE. Enhanced fat oxidation through physica activity is associated with improvements in insulin sensitivity in obesity. Diabetes 2003:52:2191-7.

10. Oza N, Eguchi $Y$, Mizuta $T$, et al. A pilot trial of body weight reduction for nonalcoholic fatty liver disease with a home-based lifestyle modification intervention delivered in collaboration with interdisciplinary medical staff. $J$ Gastroenterol 2009;44:1203-8.

11. Shah K, Stufflebam A, Hilton TN, et al. Diet and exercise interventions reduce intrahepatic fat content and improve insulin sensitivity in obese older adults. Obesity 2009;17:162-8

12. Perseghin G, Lattuada G, De Cobelli F, et al. Habitual physical activity is associated with intrahepatic fat content in humans. Diabetes Care 2007:30:683-8.

13. Zelber-Sagi S, Nitzan-Kaluski D, Goldsmith R, et al. Role of leisure-time physical activity in nonalcoholic fatty liver disease: a population-based study. Hepatology 2008:48:1791-8

14. Johnson NA, Sachinwalla T, Walton DW, et al. Aerobic exercise training reduces hepatic and visceral lipids in obese individuals without weight loss. Hepatology 2009;50:1105-12.

15. Sreenivasa Baba C, Alexander G, Kalyani B, et al. Effect of exercise and dietary modification on serum aminotransferase levels in patients with nonalcoholic steatohepatitis. J Gastroenterol Hepatol 2006;21:191-8.

16. Larose J, Sigal RJ, Boule NG, et al. Effect of exercise training on physical fitness in type II diabetes mellitus. Med Sci Sports Exerc 2010;42:1439-47.

17. Gordon BA, Benson AC, Bird SR, et al. Resistance training improves metabolic health in type 2 diabetes: a systematic review. Diabetes Res Clin Pract 2009;83:157-75

18. Angulo P, Hui JM, Marchesini G, et al. The NAFLD fibrosis score: a noninvasive system that identifies liver fibrosis in patients with NAFLD. Hepatology 2007:45:846-54.

19. St-Onge M, Mignault D, Allison DB, et al. Evaluation of a portable device to measure daily energy expenditure in free-living adults. Am J Clin Nutr 2007:85:742-9.

20. Biaggi RR, Vollman MW, Nies MA, et al. Comparison of air-displacement plethysmography with hydrostatic weighing and bioelectrical impedance analysis for the assessment of body composition in healthy adults. Am J Clin Nutr 1999;69:898-903

21. Sardinha LB, Lohman TG, Teixeira PJ, et al. Comparison of air displacement plethysmography with dual-energy $\mathrm{X}$-ray absorptiometry and 3 field methods for estimating body composition in middle-aged men. Am J Clin Nutr 1998;68:786-93.

22. Fields DA, Higgins PB, Radley D. Air-displacement plethysmography: here to stay. Curr Opin Clin Nutr Metab Care 2005;8:624-9.

23. Naressi A, Couturier C, Castang I, et al. Java-based graphical user interface for MRUI, a software package for quantitation of in vivo/medical magnetic resonance spectroscopy signals. Comput Biol Med 2001;31:269-86.

24. Naressi A, Couturier C, Devos JM, et al. Java-based graphical user interface for the MRUI quantitation package. MAGMA 2001:12:141-52.

25. Vanhamme L, Van Huffel S, Van Hecke P, et al. Time-domain quantification of series of biomedical magnetic resonance spectroscopy signals. J Magn Reson 1999:140:120-30.

26. Longo $\mathbf{R}$, Pollesello $P$, Ricci $C$, et al. Proton MR spectroscopy in quantitative in vivo determination of fat content in human liver steatosis. J Magn Reson Imaging 1995;5:281-5.
27. Donnelly LF, O'Brien KJ, Dardzinski BJ, et al. Using a phantom to compare MR techniques for determining the ratio of intraabdominal to subcutaneous adipose tissue. AJR Am J Roentgenol 2003;180:993-8.

28. Shen W, Punyanitya $M$, Wang Z, et al. Visceral adipose tissue: relations between single-slice areas and total volume. Am J Clin Nutr 2004;80:271-8.

29. Abramoff MD, Magelhaes PJ, Ram SJ. Image processing with ImageJ. Biophotonics International 2004;11:36-42.

30. Le Floch JP, Escuyer P, Baudin E, et al. Blood glucose area under the curve. Methodological aspects. Diabetes Care 1990:13:172-5.

31. Bloomgarden ZT. Measures of insulin sensitivity. Clin Lab Med 2006;26:611-33

32. Patel JV, Vyas A, Prabhakaran D, et al. Nonesterified fatty acids as mediators of glucose intolerance in Indian Asian populations. Diabetes Care 2005:28:1505-7.

33. ACSM. ACSM's Resource Manual for Guidelines for Exercise Testing and Prescription. 5th edn. Lippincott Williams and Wilkins, 2006.

34. Szczepaniak LS, Nurenberg P, Leonard D, et al. Magnetic resonance spectroscopy to measure hepatic triglyceride content: prevalence of hepatic steatosis in the general population. Am J Physiol Endocrinol Metab 2005;288:E462-8.

35. Fracanzani AL, Valenti L, Bugianesi E, et al. Risk of severe liver disease in nonalcoholic fatty liver disease with normal aminotransferase levels: a role for insulin resistance and diabetes. Hepatology 2008:48:792-8.

36. Mofrad P, Contos MJ, Haque M, et al. Clinical and histologic spectrum of nonalcoholic fatty liver disease associated with normal ALT values. Hepatology 2003;37:1286-92.

37. Petersen KF, Dufour S, Befroy D, et al. Reversal of nonalcoholic hepatic steatosis hepatic insulin resistance, and hyperglycemia by moderate weight reduction in patients with type 2 diabetes. Diabetes 2005:54:603-8.

38. Strasser B, Siebert U, Schobersberger W. Resistance training in the treatment of the metabolic syndrome: a systematic review and meta-analysis of the effect of resistance training on metabolic clustering in patients with abnormal glucose metabolism. Sports Med 2010;40:397-415.

39. Zanuso S, Jimenez A, Pugliese G, et al. Exercise for the management of type 2 diabetes: a review of the evidence. Acta Diabetol 2010;47:15-22.

40. Speliotes EK, Massaro JM, Hoffmann U, et al. Fatty liver is associated with dyslipidemia and dysglycemia independent of visceral fat: the Framingham heart study. Hepatology 2010;51:1979-87.

41. Hoenig M, Cowin G, Buckley R, et al. Liver fat percent is associated with metabolic risk factors and the metabolic syndrome in a high-risk vascular cohort. Nutr Metab 2010; 7:50

42. Lavoie J, Gauthier M. Regulation of fat metabolism in the liver: link to non-alcoholic hepatic steatosis and impact of physical exercise. Cell Mol Life Sci 2006;63:1393-409

43. Tamura Y, Tanaka Y, Sato F, et al. Effects of diet and exercise on muscle and liver intracellular lipid contents and insulin sensitivity in type 2 diabetic patients. J Clin Endocrinol Metab 2005;90:3191-6.

44. Timlin MT, Parks EJ. Temporal pattern of de novo lipogenesis in the postprandial state in healthy men. Am J Clin Nutr 2005;81:35-42.

45. Donnelly JE, Smith BK. Is exercise effective for weight loss with ad libitum diet? Energy balance, compensation, and gender differences. Exerc Sport Sci Rev 2005:33:169-74

46. Ferrannini E, Barrett EJ, Bevilacqua $S$ et al. Effect of fatty acids on glucose production and utilization in man. J Clin Invest 1983;72:1737-47.

47. Taylor R. Pathogenesis of type 2 diabetes: tracing the reverse route from cure to cause. Diabetologia 2008:51:1781-9.

48. Ferrara CM, Goldberg AP, Ortmeyer HK, et al. Effects of aerobic and resistive exercise training on glucose disposal and skeletal muscle metabolism in older men. $J$ Gerontol A Biol Sci Med Sci 2006:61:480-7.

49. Holten MK, Zacho M, Gaster M, et al. Strength training increases insulin-mediated glucose uptake, GLUT4 content, and insulin signaling in skeletal muscle in patients with type 2 diabetes. Diabetes 2004;53:294-305.

50. Pruchnic R, Katsiaras A, He J, et al. Exercise training increases intramyocellular lipid and oxidative capacity in older adults. Am J Physiol Endocrinol Metab 2004;287 E857-62.

51. Dube JJ, Amati F, Stefanovic-Racic M, et al. Exercise-induced alterations in intramyocellular lipids and insulin resistance: the athlete's paradox revisited. Am J Physiol Endocrinol Metab 2008;294:E882-8.

52. Schenk S, Horowitz JF. Acute exercise increases triglyceride synthesis in skeletal muscle and prevents fatty acid-induced insulin resistance. J Clin Invest 2007;117:1690-8.

53. Ratziu V, Bellentani $\mathrm{S}$, Cortez-Pinto $\mathrm{H}$, et al. A position statement on NAFLD/NASH based on the EASL 2009 special conference. J Hepatol 2010;53:372-84 